\title{
Spatiotemporal Quantification of Metastatic Tumour Cell Growth and Distribution in Lymph Nodes by Whole-Mount Tissue 3D Imaging
}

Zhi-Gang Zhang ( $\sim$ zzhang@shsci.org )

Shanghai Jiao Tong University https://orcid.org/0000-0001-8965-223X

Jun Li

Shanghai Jiao Tong University

Chun-Jie Xu

Shanghai Jiao Tong University

Guang-Ang Tian

Shanghai Jiao Tong University

Qing Li

Shanghai Jiao Tong University

Dong-Xue Li

Shanghai Jiao Tong University

Fang Yan

Shanghai Jiao Tong University

Xu Wang

Shanghai Jiao Tong University

Shu-Heng Jiang

Shanghai Jiao Tong University

Ya-Hui Wang

Shanghai Jiao Tong University

Yao-Qi Zhou

Shanghai Jiao Tong University

Jian Song

Shanghai Jiao Tong University

Xue-Li Zhang

Shanghai Jiao Tong University

Qing Xu

Shanghai Jiao Tong University

Li-Peng $\mathrm{Hu}$

Shanghai Jiao Tong University

Xiaowei Li 
Shanghai Jiao Tong University https://orcid.org/0000-0002-2915-1422

Article

Keywords: Spatiotemporal, metastatic tumour, lymph nodes, tissue 3D imaging

Posted Date: December 2nd, 2021

DOI: https://doi.org/10.21203/rs.3.rs-1073121/v1

License: (c) (1) This work is licensed under a Creative Commons Attribution 4.0 International License. Read Full License 


\section{Abstract}

Lymph nodes (LNs) are a common site of metastasis in many solid cancers. Tumour cells can migrate to LNs for further metastatic colonization of distant organs, indicating poor prognosis and requiring different clinical interventions. The current histopathological diagnostic methods used for the detection of clinical lymph node metastasis (LNM) still have some limitations, such as incomplete observation. To obtain a complete picture of tumour-metastasized LNs at spatial and temporal scales, we used 3D imaging of solvent-cleared organs (UDISCO) and 3D rapid immunostaining. MC38 cells tagged with EGFP were injected into the left footpad of C57BL/ 6 mice. Draining lymph nodes (DLNs) obtained from these mice were cleared using uDISCO. Metastatic colorectal cancer (CRC) cells in various regions of DLNs from mice at different time points were quantified using whole-mount tissue 3D imaging. The results revealed several stages of tumour cell growth and distribution in LNs: 1) invasion of lymphatic vessels (LVs) and blood vessels (BVs); 2) dispersion outside LVs and BVs for proliferation and expansion; and 3) re-entry into BVs and efferent lymphatic vessels (ELVs) for further distant metastasis. Moreover, these data demonstrated that mouse fibroblast cells (MFCs) could not only promote the LNM of tumour cells but could also metastasize to LNs together with tumour cells, thus providing a "soil" for tumour cell colonization. In conclusion, whole-mount tissue 3D imaging and spatiotemporal analysis of LNM may together constitute an auxiliary method to improve the accuracy of clinical LNM detection in the future.

\section{Introduction}

Emerging research has indicated that lymph nodes (LNs) are central concentrative areas for recirculating immune cells. Tumour cells can also use this gateway function of LNs for metastatic colonization of peripheral or distant organs $(1-3)$. Lymphatic dissemination is a common metastatic route of solid cancers. Metastatic tumour cells always acquire driver genetic changes during the colonization of LNs, which suggests that LNs play an important role in the evolution of cancers(4-6). The accuracy of tumour deposit quantification and lymph node metastasis (LNM) detection in solid cancers directly affects the determination of clinical tumour-node-metastasis (TNM) staging and prognostication for patients with cancer $(7,8)$. Therefore, the accurate detection and selective removal of LNs harboring metastatic tumour cells is of value for cancer diagnosis and therapy.

Until now, histopathological assessment has been the most frequently used method to assess clinical samples. Immunohistochemical (IHC) staining is one of the most commonly used methods to detect histopathological changes in clinical tissues from patients(9-13). The tissues collected for histopathological observation rely on sectioning to observe non-superficial cellular structures deep in tissues(14-16). However, traditional histopathological diagnostic methods still have some limitations in the detection of metastatic tumour cells in LNs. It is difficult to obtain accurate spatiotemporal metastatic tumour cell measurements, including the number, localization and morphology of these cells in intact tissues. 
In recent years, the investigation of complex cells with large projections has been best performed in intact tissue. Recently developed tissue-clearing methods have overcome obstacles imposed by light scattering(17-19). Ultimate 3D imaging of solvent-cleared organs (UDISCO) is a method that was developed to reveal and preserve endogenous fluorescence signal over months while retaining the advantageous size reduction $(14,20)$. Currently, in various biomedical applications, uDISCO has been used to study the organization of large organ systems. Furthermore, a 3D rapid immunostaining system was developed to optimize the process of antibody labelling in intact tissues(21). Using this system, the labelling time of millimetre-thick cleared specimens can be shortened from weeks to hours.

In this study, colorectal cancer (CRC)-related LNM was investigated by constructing a mouse footpad model in which CRC cells were injected into the left footpad of mice. The uDISCO method was used to clear and observe lymph node ( $L N)$ tissues. Co-metastasis of CRC cells and fibroblasts was also detected by whole-mount tissue 3D imaging. The accurate spatiotemporal quantification of metastatic tumour cells in LNs detected by whole-mount tissue 3D imaging may potentially improve the accuracy of clinical LNM detection and could complement traditional pathological methods of clinical histopathological diagnosis.

\section{Results}

\section{Whole-mount tissue 3D imaging of draining lymph nodes and quantification of invaded tumour cells.}

The mouse model of LNM was constructed using a CRC cell line. MC38 cells tagged with enhanced green fluorescent protein (EGFP) were injected into the left footpad of C57BL/6 mice, and the fluorescence intensity was tracked and detected using a Bio-Real in vivo imaging system (Supplementary Fig. 1). The draining lymph nodes (DLNs) were collected every five days for a total duration of 30 days (Fig. 1a). The dissected DLNs were fixed in 4\% PFA and then cleared using the uDISCO method (Fig. 1b). The cleared DLNs maintained both endogenous fluorescence signal and the advantageous size reduction (Fig. 1C). Moreover, we found that the effect of LYVE-1 immunostaining performed by 3D rapid immunostaining was similar to that of the traditional immunostaining method (Supplementary Fig. 2).

To accurately quantify the tumour cells, the cleared DLNs were imaged using confocal microscopy. Because tumour cells were found too dense in some areas and difficult to distinguish single cells, we used the tumour cell-associated pixel number instead of the number of tumour cells. By comparing the pixel number and the number of tumour cells in a randomly selected area, we found that the tumour cellassociated pixel number can directly reflect the number of tumour cells. In each group, we calculated the tumour cell-associated pixel number in the LN image at each time point. After all the time points in a group were calculated, the tumour cell-associated pixel number in the LNs from a particular group of images was obtained. All groups were analysed in the same way to allow for inter-group comparisons. The total invaded MC38 cell-associated pixel number in all DLNs at different time points was quantified using Imaris image processing software (Figure 1d, Supplementary Video 1-4). The number of invaded EGFP-tagged MC38 cells gradually increased in DLNs at $5 \mathrm{~d}, 10 \mathrm{~d}, 20 \mathrm{~d}$ and $30 \mathrm{~d}$ (Fig. 1e). 


\section{The spatiotemporal distribution of metastatic CRC cells in DLNs.}

Previous studies have reported the distribution of different lymphatic vessels (LVs) and blood vessels (BVs) in LNs(22), including afferent lymphatic vessels (ALVs), efferent lymphatic vessels (ELVs), high endothelial venules (HEVs) and arteries (Fig. 2a). Here, we confirmed the distribution of LVs and BVs in the DLNs by IHC staining (Fig. 2b). Then, using whole-mount tissue 3D imaging, whole LN tissues were immunostained with antibodies against LYVE-1 (for LVs) and CD31 (for BVs). Colocalization of LYVE1 and EGFP signals indicated MC38 cells distributed in ELVs (e-MC38), colocalization of CD31 and EGFP signals indicated MC38 cells distributed in BVs (b-MC38), and EGFP signal alone indicated MC38 cells scattered outside lymphatic/blood vessels (s-MC38) (Fig. 2c-e). MC38 cells and associated pixel number were observed and analyzed by using Imaris (Fig. 2 f and Fig. 3c, d).

After UDISCO clearing, the distribution of EGFP-tagged MC38 cells in the different regions of DLNs was observed by 3D imaging (Fig. 3a, b). We then quantified the tumour cell-associated pixel number in different regions of DLNs. The results showed that the pixel number associated with MC38 cells distributed in ELVs or BVs was unchanged between days 5 and 20 but significantly increased from day 20 to 30 . Meanwhile, the pixel number associated with MC38 cells scattered outside lymphatic/blood vessels significantly increased from day 5 to 10 . However, no changes were observed in the pixel number associated with scattered tumour cells at time points beyond day 10 (Fig. 3c).

Interestingly, a relatively high percentage of MC38 cells were characterized as e-MC38 cells $(51.89 \%)$ or bMC38 cells (24.16\%) at the early time point of day 5 . At days 10 and 20, the ratio of e-MC38/total MC38 cells was found to be decreased $(33.19 \%$ and $21.51 \%$, respectively). Similar changes were also observed in the ratio of b-MC38/total MC38 cells at day 10 (13.55\%) and day $20(26.59 \%)$. At the last time point (day 30), a significant increase was observed in the ratio of e-MC38/total MC38 cells (33.44\%) and bMC38/total MC38 cells (50.72\%). However, the opposite trend was observed for s-MC38 cells, where the sMC38/total MC38 cell ratio was relatively low (23.95\%) at day 5 and subsequently increased to $53.26 \%$ (day 10) and $51.90 \%$ (day 20) and finally decreased to $15.84 \%$ (day 30) (Fig. 3a, b). At least 3 individual samples in each group were detected and are shown as a statistical result in Fig. 3d.

According to the statistical results, the distribution of metastatic tumour cells was concluded. Our results demonstrated that metastatic tumour cells mainly invaded ELVs and BVs in the early stage of LNM. Then, most of these cells moved and scattered outside LVs or BVs for proliferation and expansion. Finally, tumour cells reaggregated in BVs and ELVs to support further metastasis (Fig. 3e). We also showed that spatiotemporal information of metastatic tumour cells in LNs can be accurately obtained using wholemount tissue 3D imaging.

\section{The proliferation and expansion of metastatic CRC cells in DLNs.}

To further confirm the outgrowth of MC38 cells in different LN regions, we used IHC staining for Ki67 to observe the proliferation of metastatic CRC cells in DLNs (Fig. 4a-d). LYVE-1-positive areas (LVs) were stained in red, and CD31-positive areas (BVs) were stained in purple. Then, we superimposed the red and 
purple areas on the third serial section to identify metastatic tumour cells distributed in or outside the lymphatic/blood vessels. Total Ki67 (t-Ki67)-positive cells were characterized as the tumour cells distributed in the whole LN, and scattered Ki67 (s-Ki67)-positive cells were characterized as the tumour cells distributed outside lymphatic/blood vessels.

By calculating the number of proliferating tumour cells in different regions, we found that both t-Ki67positive cells and s-Ki67-positive cells increased with time. However, the rate at which s-Ki67-positive cells increased over time diminished between days 20 and 30, while the number of Ki67 cells distributed in lymphatic and blood vessels increased significantly during this last time period (Fig. 4e-g). All of the above data further confirmed the spatiotemporal information obtained from whole-mount tissue 3D imaging.

\section{The co-metastasis of CRC cells and mouse fibroblast cells in DLNs.}

Recent reports have indicated that fibroblasts provide a "soil" for metastatic tumour cells. We therefore investigated the effects of fibroblasts on CRC cells that disseminated to LN tissues by using whole-mount tissue 3D imaging. MC38 cells tagged with EGFP and mouse fibroblast cells (MFCs) tagged with mCherry were cocultured or mixed together and divided into three groups: vehicle, coculture and mixed. In the coculture group, MC38 cells and MFCs were mixed and incubated for 24 hours and then injected into the left footpad of mice. In the mixed group, MC38 cells or MFCs were mixed together immediately prior to injection into the left footpad of mice (Fig. 5a).

DLNs were obtained and analysed after $5 \mathrm{~d}$ and $10 \mathrm{~d}$. DLNs obtained from the mixed or cocultured group were significantly larger in volume compared with those in the vehicle group at $5 \mathrm{~d}$ and $10 \mathrm{~d}$ (Fig. 5b, c). DLNs were cleared using uDISCO to analyse infiltrating tumour cells. At days 5 and 10 , the number of metastatic CRC cells was significantly greater in the mixed and cocultured groups compared with the vehicle group, which suggested that MFCs could promote the metastasis of CRC cells (Fig. $5 \mathrm{~d}-\mathrm{g}$ ). MFCs were also found to be scattered in LNs at $5 \mathrm{~d}$ and $10 \mathrm{~d}$, where they grew together with CRC cells. The metastatic MFCs in LN tissues provide a "soil" for CRC cells, thus supporting the metastasis and growth of CRC cells in the LNs (Fig. 5d-g).

\section{Whole-mount tissue 3D imaging of mesenteric LN tissues from clinical CRC patients.}

To apply whole-mount tissue 3D imaging in clinical research, mesenteric LN tissues were collected from CRC patients according to a standard procedure, fixed in 4\% PFA, and cleared using uDISCO (Fig. 6a). Cleared LN tissue collected from a 70-year-old man retained the advantageous size reduction (Fig. 6b). After immunostaining with an antibody against CK-20 (for CRC cells) and the nuclear dye TO-PRO-3 (for nuclei), we observed the cleared lymph nodes by laser scanning confocal microscopy. CRC cells labelled with CK-20 were observed and are shown in fields 1 and 2, and unstained cells are shown in field 3 (Fig. 6c, d). 
Whole-mount tissue 3D imaging was used in conjunction with Imaris 9.0 software to photograph and process the LN tissue. The whole LN was shown as a reconstructed 3D image (Fig. 6e). Invasion of CRC cells can be clearly observed in the LN cross-section (Fig. 6f). Whole-mount tissue 3D imaging may be used together with traditional histopathological methods to improve the accuracy of clinical LNM detection and aid in clinical diagnosis.

\section{Discussion}

LNs are complex organs that harbour multiple specialized stromal, myeloid, and lymphoid cells(23). LNs are composed of well-developed smooth muscles under a capsular layer, and the lymph flowing in from the ALVs is squeezed out by the capsular smooth muscles into the ELVs. Under normal conditions, the valves of ALVs and ELVs prevent retrograde flow $(24,25)$. Previous studies have indicated that tumour cells metastasize to LNs by reconstructing the local immune system and transforming immune cells, but spatiotemporal changes in tumour cells in whole-mount LNs have not been reported $(2,3,26)$. In this study, we used whole-mount tissue 3D imaging to accurately quantify the number of metastatic CRC cells in DLNs of mice at different time points and in different $L N$ regions. The traditional immunostaining method used in uDISCO clearing requires 1 to 2 weeks for antibody labelling. Here, a 3D rapid immunostaining system was used to shorten the antibody labelling time from weeks to hours for intact LNs during the process of uDISCO. We found that tumour cells enter the LN through ALVs but then proliferate outside of the lymphatic and blood vessels, potentially due to the limited space within these vessels. When the tumour cells in LNs proliferate to a certain number, most of them migrate back into BVs and ELVs to facilitate their further distant metastasis. By whole-mount tissue 3D imaging, we can obtain both spatial and temporal information regarding the distribution of metastatic tumour cells in LNs, providing a theoretical basis for further understanding the metastatic biological characteristics of tumour cells in LNs.

At present, the clinical detection of CRC-related LNs mainly depends on traditional histopathological methods. Intestinal planar and distal LNs are detected by observing CK7, CK20, CDX2 or other markers(27-29). Because these histopathological methods rely on imaging in two-dimensional tissue sections, the information obtained is limited. In a recent study, over 280,000 slices of an entire murine LN tissue were captured by an extended-volume imaging system to observe the conduit network in the whole tissue(30). In this study, we aimed to use a simpler method to achieve a comprehensive observation of whole LN tissue and to effectively obtain the spatial and temporal information of metastatic tumour cells in LNs. Using whole-mount tissue 3D imaging and a 3D rapid immunostaining system for intact tissues, we obtained spatiotemporal information for metastatic tumour cells in LNs and reduced the total time required for the clearing and staining process to 3 days. In the future, this method may play an auxiliary role in the clinical detection of solid cancer-related LNM.

Reports in recent years have indicated that tumour cells metastasize to LNs very early in cancer progression. During the process of tumour cell-related LNM, tumour cells undergo driver genetic changes while stromal and immune cells respond to promote the formation of a "premetastatic niche" in LNs, thus 
forming a tumour microenvironment for the colonization and growth of tumour cells in $\operatorname{LNs}(4,6)$. It has been reported that Siglec $1^{+}$subcapsular sinus macrophages can provide a "soil" for the LNM of melanoma cells(6). Here, we observed the co-metastasis of fibroblasts and tumour cells in LNs by wholemount tissue 3D imaging. Interestingly, MFCs were able to promote tumour cell LNM but could also metastasize to LNs together with tumour cells, thus providing a "soil" for tumour cell colonization. In future, we will further screen the key molecules that play important roles in the regulatory effects of MFCs on the metastasis and colonization of tumour cells, and uncover the potential underlying mechanism. 3D imaging was used to reveal this process and may serve as a new method to study the co-metastasis of tumour and stromal cells.

In conclusion, whole-mount tissue 3D imaging can provide accurate spatiotemporal quantification of metastatic tumour cells in LNs, which may complement traditional histopathological methods of clinical diagnosis and could affect clinical TNM stage determination for patients with cancer. In the future, we may further optimize and combine whole-mount tissue 3D imaging, 3D rapid immunostaining and spatiotemporal quantification as an auxiliary method to improve the accuracy of clinical LNM detection.

\section{Declarations}

\section{Acknowledgements}

The authors thank Prof. Xue-Yun Ma, Dr. Yan-Li Zhang, Dr. Xiao-Mei Yang, Dr. Hui-Zhen Nie, Dr. Lei Zhu, Dr. Lin-Li Yao, Dr. Wei-Ting Qin, Ms. M. Kuerbanjiang, Ms. Yan Wang, Ms. Dong-Ming Yang, Dr. Ni Zhang, Dr. Li-li Zhu, Dr. Shan Huang, Dr. Shan Zhang, Ms. Pei-Qi Huang and Ms. Kai-Xia Zhou for assistance with our experiments.

\section{Author contributions}

J.L. and Z.-G.Z. designed and supervised the overall study, analyzed data, and drafted the manuscript. J.L., L.-P.H. and X.-L.Z. constructed the mouse footpad model and collected the LN tissues. J.L., C.-J.X., G.-A.T. and D.-X.L. performed the uDISCO clearing and whole-mount tissue 3D imaging. Q.L. and X.W. assisted with analysis of immunohistochemical staining. F.Y. analyzed the data of 3D imaging. S.-H.J., Y.H.W., Y.-Q.Z. and J.S. technically assisted with experiments and analyzed data. Q.X. provided human LN tissues. Q.X., L.-P.H. and X.-W.L. supervised this study and edited the manuscript.

\section{Funding}

This work was supported by the National Natural Science Foundation of China (ID 82073023, 81871923, to J. Li; ID 81802890, to X.L. Zhang; ID 82002485, to Q. Li; ID 31801212, to L.L. Yao; ID 31970745 to J. Song), the Shanghai Municipal Education Commission-Gaofeng Clinical Medicine Grant Support (ID 20191809, to J. Li; ID 20181708, to Z.G. Zhang), Program of Shanghai Academic/Technology Research Leader (ID 19XD1403400, to Z.G. Zhang), Shanghai International Science and Technology Cooperation Fund (ID 18410721000, to Z.G. Zhang) and Excellent Academic Leader of Shanghai Municipal Health 
Bureau (ID 2018BR32, to Z.G. Zhang), Medicine and Engineering Interdisciplinary Research Fund of Shanghai Jiao Tong University (ID YG2021ZD08, to Z.G. Zhang), the Shanghai Municipal Health Commission (ID 202040092 to X.L. Zhang).

\section{Competing interests}

The authors declare no competing interests.

\section{References}

1. Brown M, Assen FP, Leithner A, Abe J, Schachner H, Asfour G, et al. Lymph node blood vessels provide exit routes for metastatic tumor cell dissemination in mice. Science. 2018;359:1408-11.

2. Reynoso GV, Weisberg AS, Shannon JP, McManus DT, Shores L, Americo JL, et al. Lymph node conduits transport virions for rapid T cell activation. Nature immunology. 2019;20:602-12.

3. Pereira ER, Kedrin D, Seano G, Gautier O, Meijer EFJ, Jones D, et al. Lymph node metastases can invade local blood vessels, exit the node, and colonize distant organs in mice. Science. 2018;359:1403-7.

4. Werner-Klein M, Scheitler S, Hoffmann M, Hodak I, Dietz K, Lehnert P, et al. Genetic alterations driving metastatic colony formation are acquired outside of the primary tumour in melanoma. Nature communications. 2018;9:595.

5. Lee CK, Jeong SH, Jang C, Bae H, Kim YH, Park I, et al. Tumor metastasis to lymph nodes requires YAP-dependent metabolic adaptation. Science. 2019;363:644-9.

6. Singh R, Choi BK. Siglec1-expressing subcapsular sinus macrophages provide soil for melanoma lymph node metastasis. eLife. 2019;8.

7. Song YX, Gao P, Wang ZN, Liang JW, Sun Z, Wang MX, et al. Can the tumor deposits be counted as metastatic lymph nodes in the UICC TNM staging system for colorectal cancer? PloS one. 2012;7:e34087.

8. Tong LL, Gao P, Wang ZN, Song YX, Xu YY, Sun Z, et al. Is the seventh edition of the UICC/AJCC TNM staging system reasonable for patients with tumor deposits in colorectal cancer? Annals of surgery. 2012;255:208-13.

9. Beaton C, Twine CP, Williams GL, Radcliffe AG. Systematic review and meta-analysis of histopathological factors influencing the risk of lymph node metastasis in early colorectal cancer. Colorectal disease: the official journal of the Association of Coloproctology of Great Britain and Ireland. 2013;15:788-97.

10. Wada H, Shiozawa M, Katayama K, Okamoto N, Miyagi Y, Rino Y, et al. Systematic review and metaanalysis of histopathological predictive factors for lymph node metastasis in $\mathrm{T} 1$ colorectal cancer. Journal of gastroenterology. 2015;50:727-34.

11. Yasue C, Chino A, Takamatsu M, Namikawa K, Ide D, Saito S, et al. Pathological risk factors and predictive endoscopic factors for lymph node metastasis of T1 colorectal cancer: a single-center 
study of 846 lesions. Journal of gastroenterology. 2019;54:708-17.

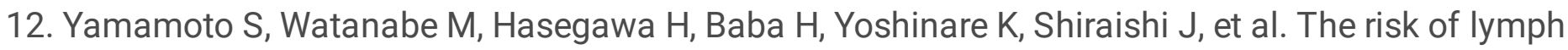
node metastasis in T1 colorectal carcinoma. Hepato-gastroenterology. 2004;51:998-1000.

13. Tateishi Y, Nakanishi Y, Taniguchi H, Shimoda T, Umemura S. Pathological prognostic factors predicting lymph node metastasis in submucosal invasive (T1) colorectal carcinoma. Modern pathology: an official journal of the United States and Canadian Academy of Pathology, Inc. 2010;23:1068-72.

14. Pan C, Cai R, Quacquarelli FP, Ghasemigharagoz A, Lourbopoulos A, Matryba P, et al. Shrinkagemediated imaging of entire organs and organisms using uDISCO. Nature methods. 2016;13:859-67.

15. Mullen R, Purdie CA, Jordan LB, McLean D, Whelehan P, Vinnicombe S, et al. Can additional histopathological examination of ultrasound-guided axillary lymph node core biopsies improve preoperative diagnosis of primary breast cancer nodal metastasis? Clinical radiology. 2013;68:7047.

16. Nowikiewicz T, Zegarski W, Pagacz K, Nowacki M, Morawiec-Sztandera A, Glowacka-Mrotek I, et al. The current application of ACOSOG Z0011 trial results: Is further implementation of sentinel lymph node intra-operative histopathological examination mandatory in breast cancer patients - a singlecentre analysis. Neoplasma. 2018;65:449-54.

17. Tainaka K, Kubota SI, Suyama TQ, Susaki EA, Perrin D, Ukai-Tadenuma M, et al. Whole-body imaging with single-cell resolution by tissue decolorization. Cell. 2014;159:911-24.

18. Erturk A, Mauch CP, Hellal F, Forstner F, Keck T, Becker K, et al. Three-dimensional imaging of the unsectioned adult spinal cord to assess axon regeneration and glial responses after injury. Nature medicine. 2011;18:166-71.

19. Yang B, Treweek JB, Kulkarni RP, Deverman BE, Chen CK, Lubeck E, et al. Single-cell phenotyping within transparent intact tissue through whole-body clearing. Cell. 2014;158:945-58.

20. Erturk A, Becker K, Jahrling N, Mauch CP, Hojer CD, Egen JG, et al. Three-dimensional imaging of solvent-cleared organs using 3DISCO. Nature protocols. 2012;7:1983-95.

21. Li J, Czajkowsky DM, Li X, Shao Z. Fast immuno-labeling by electrophoretically driven infiltration for intact tissue imaging. Scientific reports. 2015;5:10640.

22. Gasteiger G, Ataide $M$, Kastenmuller W. Lymph node - an organ for T-cell activation and pathogen defense. Immunological reviews. 2016;271:200-20.

23. Krishnamurty AT, Turley SJ. Lymph node stromal cells: cartographers of the immune system. Nature immunology. 2020;21: 369-80.

24. Akita S, Yamaji Y, Kuriyama M, Tokumoto H, Ishigaki T, Tezuka T, et al. Intraoperative Detection of Efferent Lymphatic Vessels Emerging from Lymph Node during Lymphatic Microsurgery. Journal of reconstructive microsurgery. 2019;35:372-8.

25. Ohtani O, Ohtani Y. Recent developments in morphology of lymphatic vessels and lymph nodes. Annals of vascular diseases. 2012;5:145-50. 
26. Gu Y, Liu YF, Fu L, Zhai LL, Zhu J, Han YM, et al. Tumor-educated B cells selectively promote breast cancer lymph node metastasis by HSPA4-targeting IgG. Nature medicine. 2019;25:312-+.

27. Shin JH, Bae JH, Lee A, Jung CK, Yim HW, Park JS, et al. CK7, CK20, CDX2 and MUC2 Immunohistochemical staining used to distinguish metastatic colorectal carcinoma involving ovary from primary ovarian mucinous adenocarcinoma. Japanese journal of clinical oncology. 2010;40:208-13.

28. Perysinakis I, Minaidou E, Leontara V, Mantas D, Sotiropoulos GC, Tsipras H, et al. Differential Expression of beta-Catenin, EGFR, CK7, CK20, MUC1, MUC2, and CDX2 in Intestinal and Pancreatobiliary-Type Ampullary Carcinomas. International journal of surgical pathology. 2017;25:31-40.

29. Friederichs J, Gertler R, Rosenberg R, Dahm M, Nekarda H, Holzmann B, et al. Correlation of CK-20positive cells in peripheral venous blood with serum CEA levels in patients with colorectal carcinoma. World journal of surgery. 2007;31:2329-34.

30. Kelch ID, Bogle G, Sands GB, Phillips ARJ, LeGrice IJ, Dunbar PR. High-resolution 3D imaging and topological mapping of the lymph node conduit system. PLoS biology. 2019;17:e3000486.

\section{Figures}


a

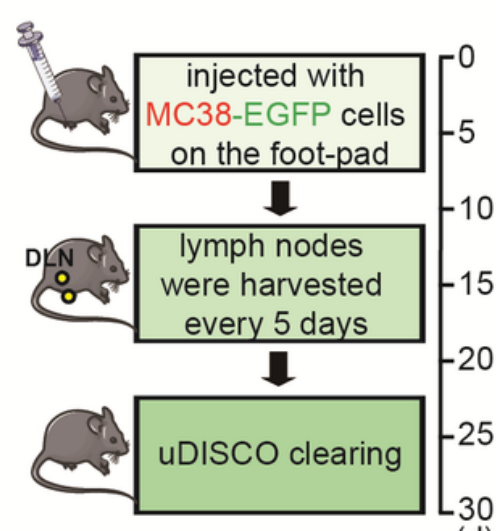

(d) b

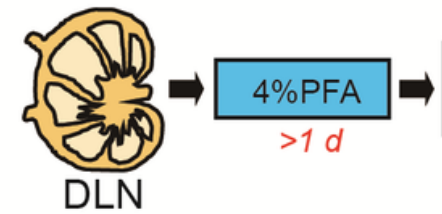

each step $1 \mathrm{~h}$

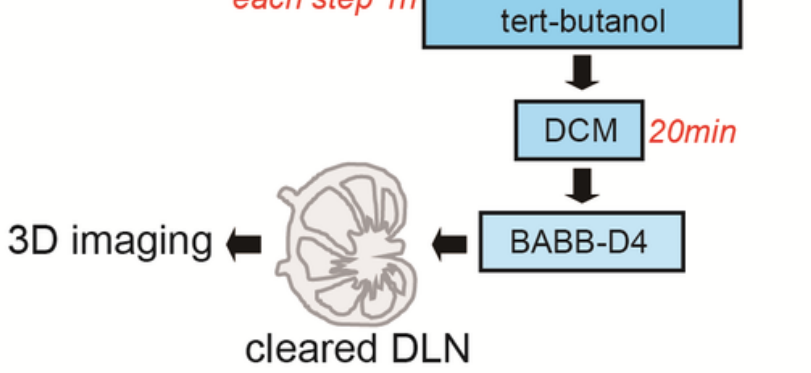

C

lymph node

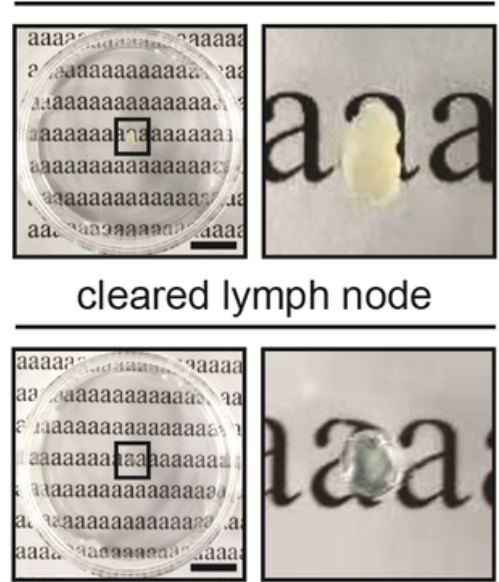

d whole-mount tissue 3D imaging

e

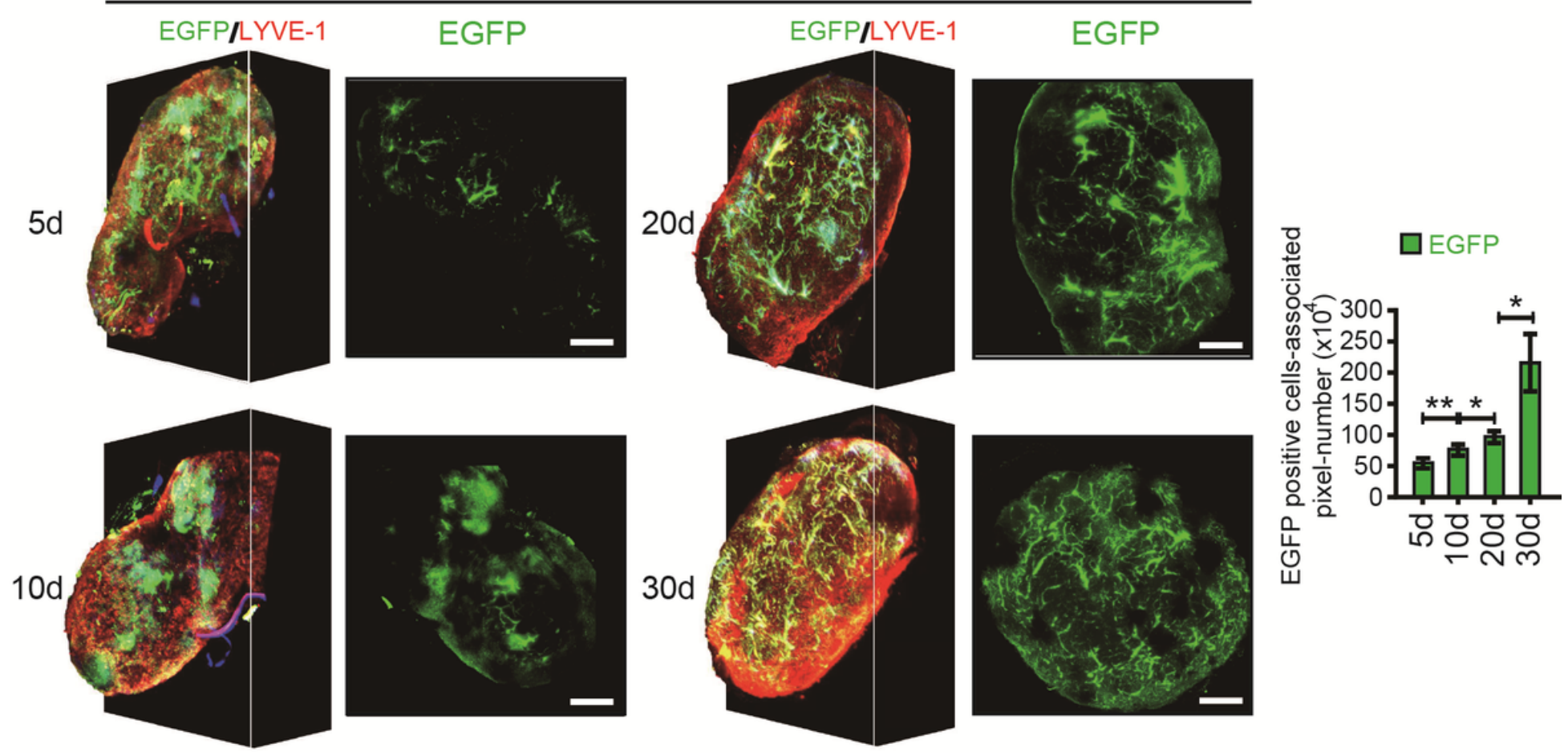

Figure 1

The whole-mount tissue 3D imaging of DLNs and quantification of tumour cells. a. The schedule of mouse footpad model construction and the time points of LN tissues collection. b. The uDISCO protocols of DLN. c. Photos of LN tissues before and after uDISCO clearing. Scale bars: $1200 \mu \mathrm{m}$. d. 3D images and cross-section photos of DLNs at the time points of $5 \mathrm{~d}, 10 \mathrm{~d}, 20 \mathrm{~d}$ and $30 \mathrm{~d}$ respectively. Scale bars: 200 $\mu \mathrm{m}$. e. The invaded EGFP-tagged MC38 cells-associated pixel-number in DLNs at the time points of $5 \mathrm{~d}$, $10 \mathrm{~d}, 20 \mathrm{~d}$ and $30 \mathrm{~d}$ quantified by Imaris. All experiments were repeated at least three times. 
a

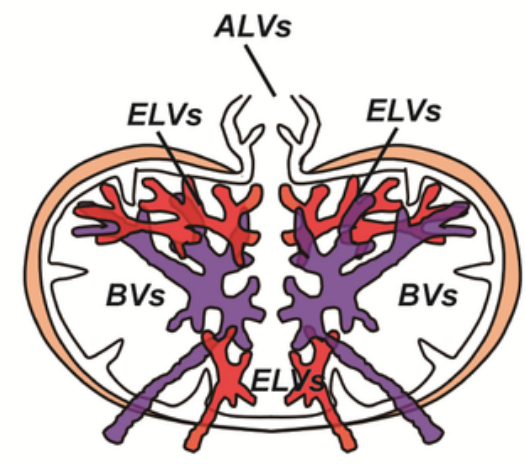

C

Scattered MC38 cells

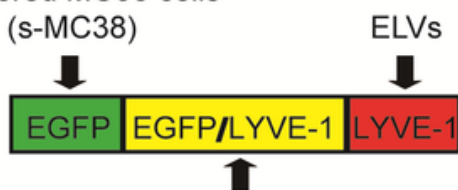

MC38 cells distributed in lymphatic vessels (e-MC38) b

LYVE-1
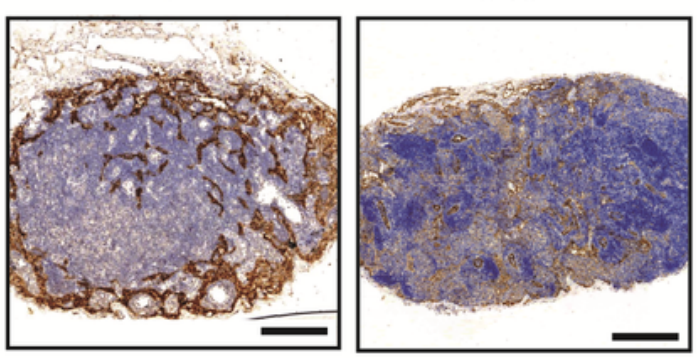

Scattered MC38 cells

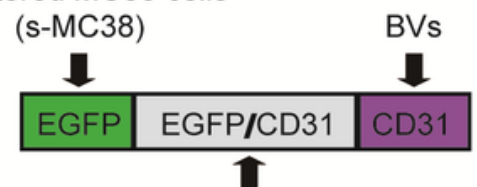

MC38 cells distributed in blood vessels (b-MC38)

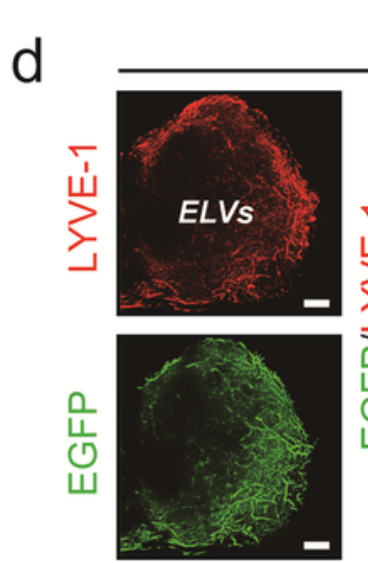

whole-mount tissue 3D imaging

e
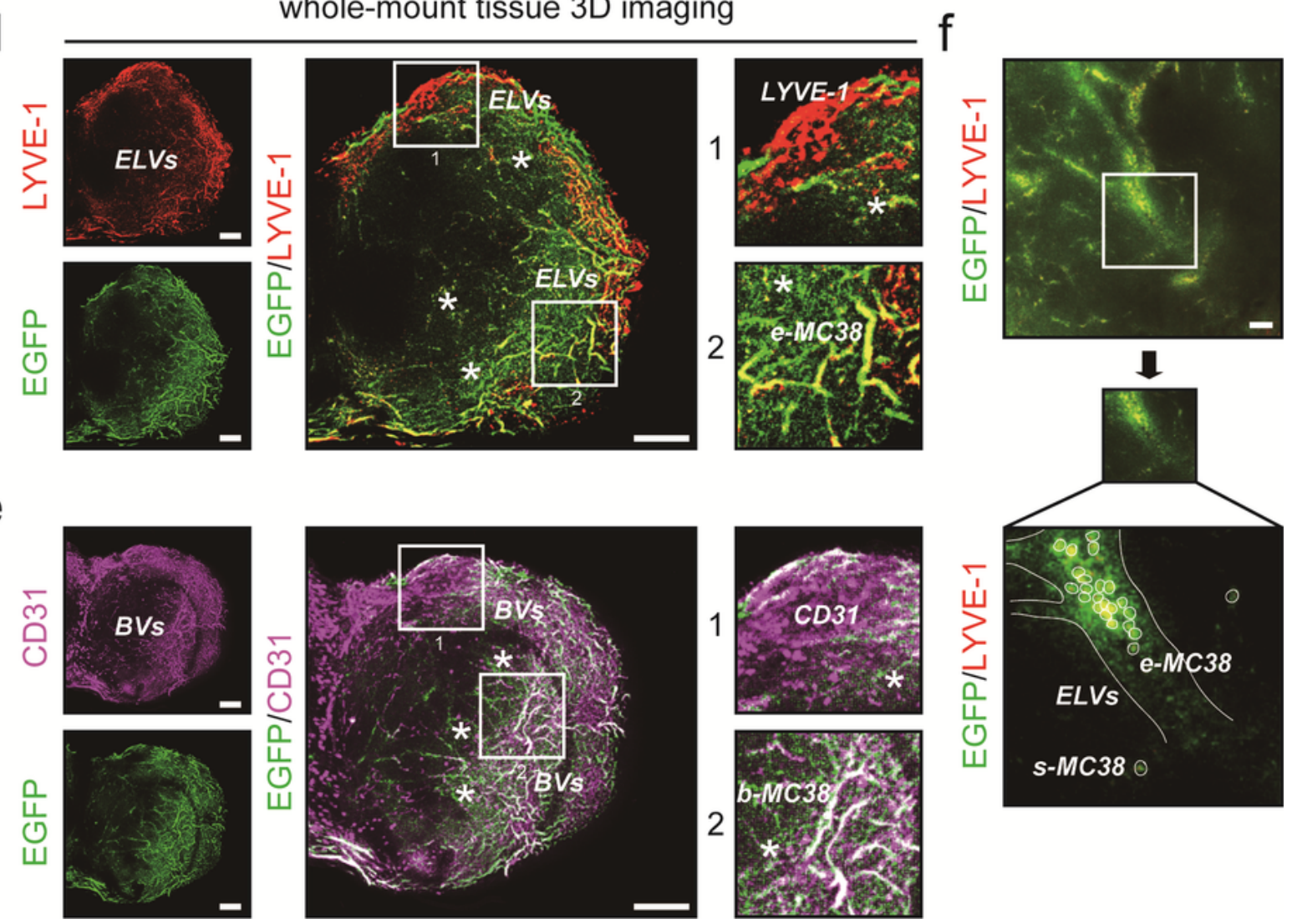

Figure 2

The immunofluorescent detection of ELVs, BVs and EGFP-tagged MC38 cells. a. Distribution of ALVs, ELVs and BVs, including HEVs and arteries in LN. b. IHC staining photos of LVs and BVs in LNs. Scale bars: $200 \mu \mathrm{m}$. c. The immunofluorescent detection of LYVE-1 (LVs) and CD31 (BVs). Colocalization of LYVE1 and EGFP signals indicated MC38 cells distributed in ELVs (e-MC38), colocalization of CD31 and EGFP signals indicated MC38 cells distributed in BVs (b-MC38), and EGFP signal alone indicated MC38 
cells scattered outside lymphatic/blood vessels (s-MC38). d, e. Cross-section photos of EGFP-tagged MC38 cells, LYVE-1 (d) and CD31 (e) in a LN at 5 d. LYVE-1: red; CD31: purple; *: MC38 cells scattered outside lymphatic/blood vessels. Scale bars: $100 \mu \mathrm{m}$. f. Cross-section photos of MC38 cells distributed in ELVs and scattered outside lymphatic/blood vessels. Scale bars: $40 \mu \mathrm{m}$.

a whole-mount tissue 3D imaging

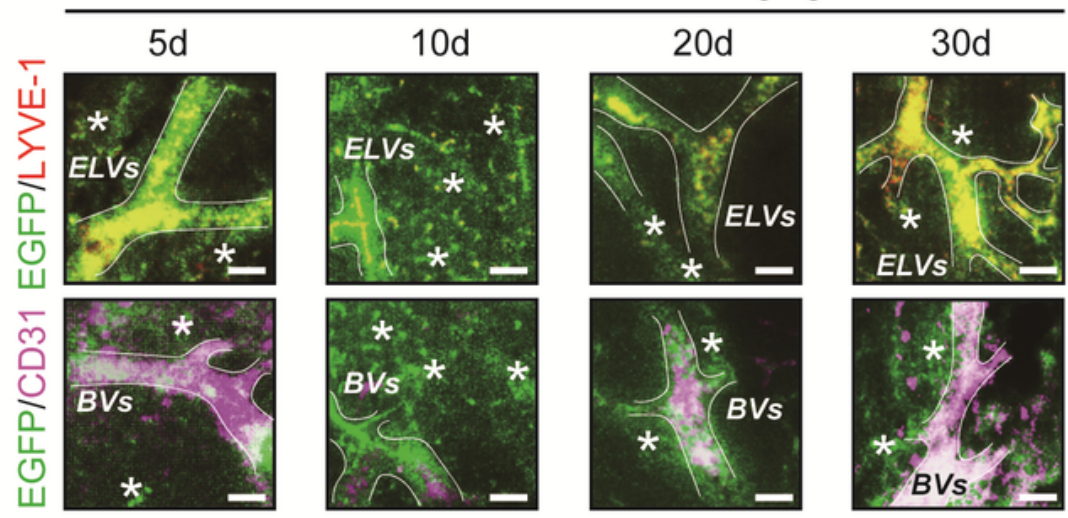

b
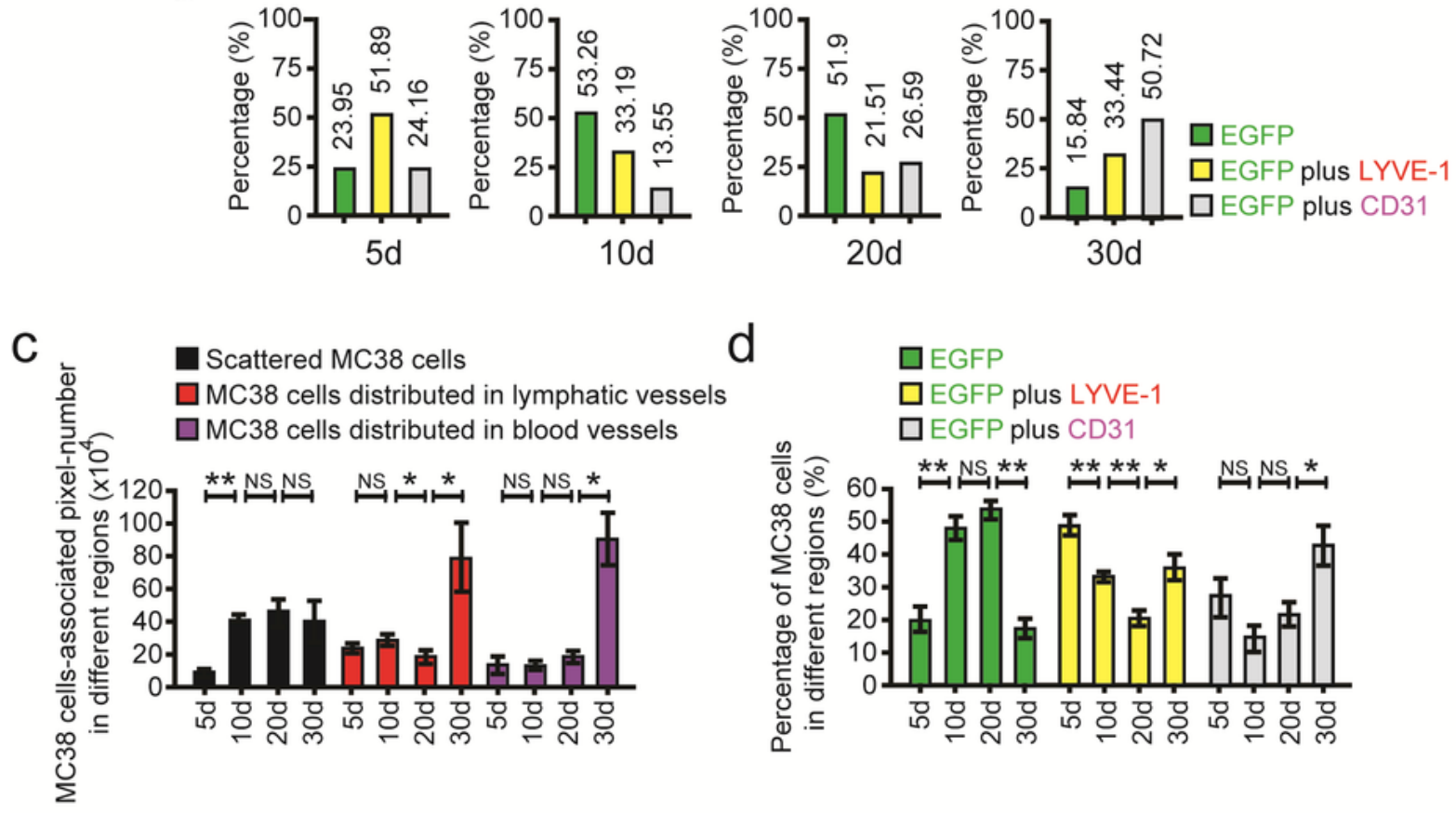

e
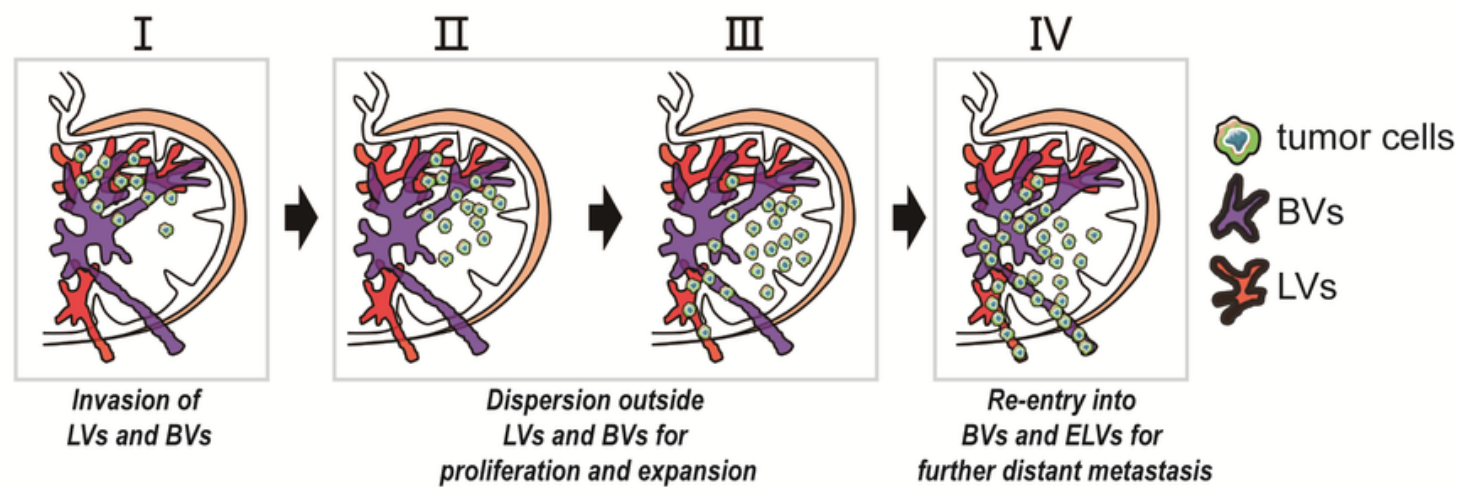

Figure 3 
The spatiotemporal quantification of metastatic CRC cells in DLNs. a, b. Cross-section photos of EGFPtagged MC38 cells in the different regions of LNs at $5 \mathrm{~d}, 10 \mathrm{~d}, 20 \mathrm{~d}$ and $30 \mathrm{~d}$ respectively (a). The percentages of MC38 cells distributed in ELVs or BVs / total MC38 cells were shown below (b). LYVE-1: red; CD31: purple; *: MC38 cells scattered outside lymphatic/blood vessels. Scale bars: $40 \mu \mathrm{m}$. c. The pixel-number associated with MC38 cells distributed in ELVs or BVs and the pixel-number associated with MC38 cells scattered outside lymphatic/blood vessels at $5 \mathrm{~d}, 10 \mathrm{~d}, 20 \mathrm{~d}$ and $30 \mathrm{~d}$ respectively. One-way ANOVA was used for comparison between groups. All experiments were repeated at least three times. $d$. The ratio of MC38 cells distributed in ELVs or BVs / total MC38 cells and the ratio of MC38 cells scattered outside lymphatic/blood vessels / total MC38 cells at $5 \mathrm{~d}, 10 \mathrm{~d}, 20 \mathrm{~d}$ and $30 \mathrm{~d}$ respectively. One-way ANOVA was used for comparison between groups. All experiments were repeated at least three times. e. The metastatic tumour cells were found mainly invaded in ELVs and BVs in the early stage of LNM, then most of them moved and scattered outside lymphatic/blood vessels in LNs for proliferation and expansion, and at last reaggregated in BVs and ELVs to support metastasis. 


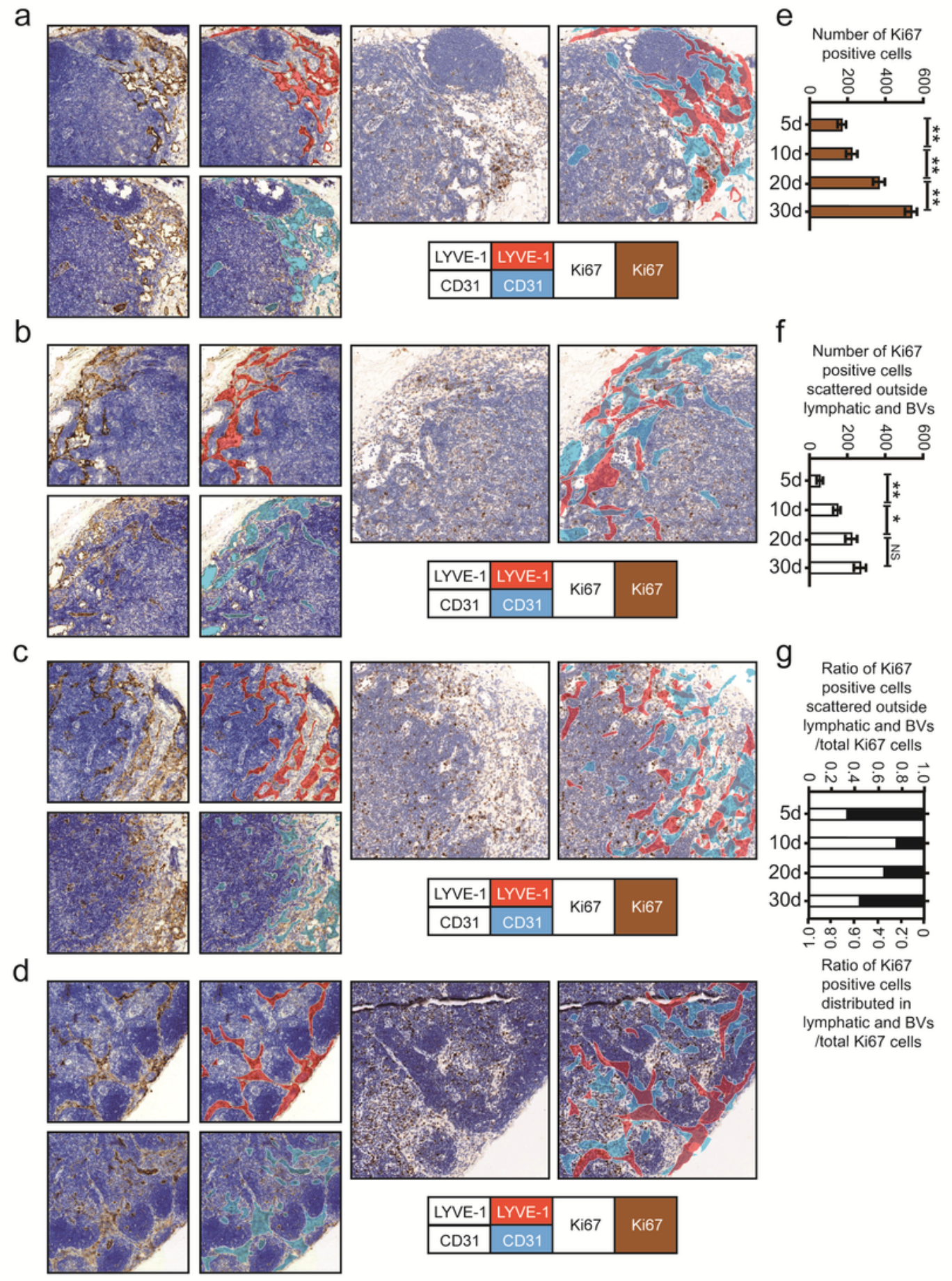

\section{Figure 4}

The proliferation of metastatic CRC cells in DLNs. a-d. IHC staining photos of Ki67, CD31 and LYVE-1 in DLNs at $5 \mathrm{~d}(\mathrm{a}), 10 \mathrm{~d}$ (b), $20 \mathrm{~d}$ (c) and $30 \mathrm{~d}$ (d). Scale bars: $100 \mu \mathrm{m}$. e, f. The statistical results of total Ki67 (e) and scattered Ki67 (f) (the tumour cells distributed outside lymphatic/blood vessels) positive cells at $5 \mathrm{~d}, 10 \mathrm{~d}, 20 \mathrm{~d}$ and $30 \mathrm{~d}$ respectively. All experiments were repeated at least three times. $\mathrm{g}$. The 
ratio of Ki67 positive cells (scattered outside lymphatic/blood vessels) / total Ki67 cells and Ki67 positive cells (distributed in lymphatic/blood vessels) / total Ki67 cells at 5 d, 10 d, 20 d and $30 \mathrm{~d}$ respectively.

a
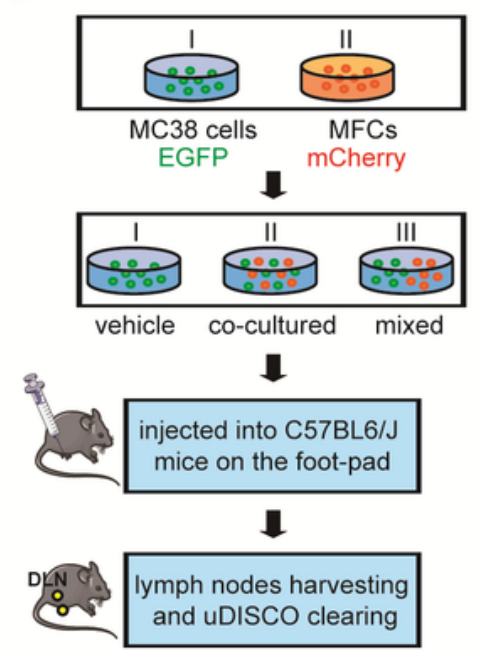

d
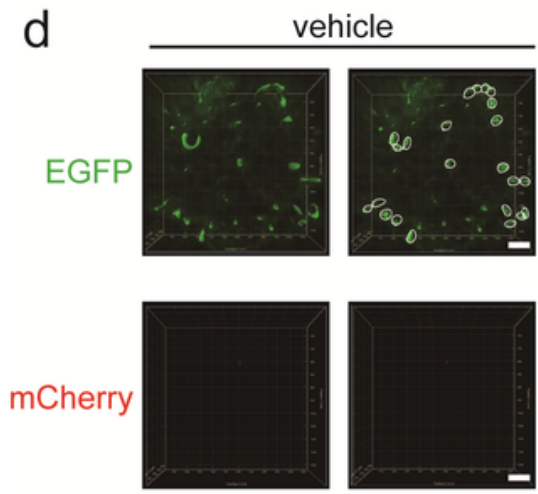

e

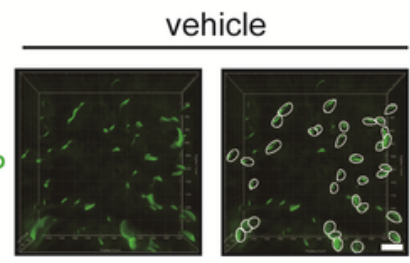

mCherry

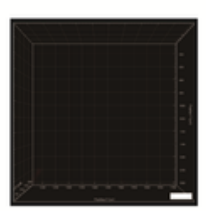

b

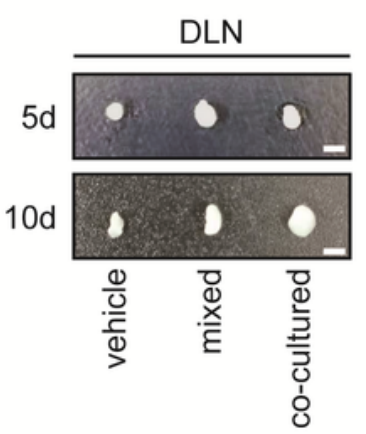

C

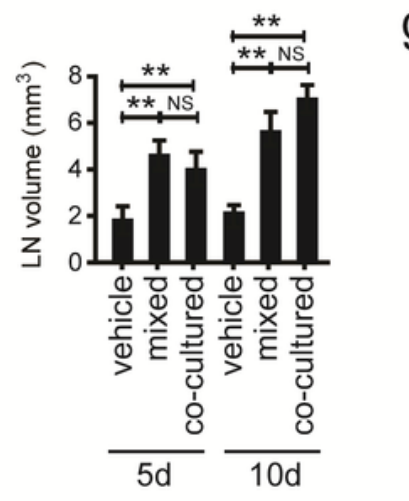

whole-mount tissue 3D imaging
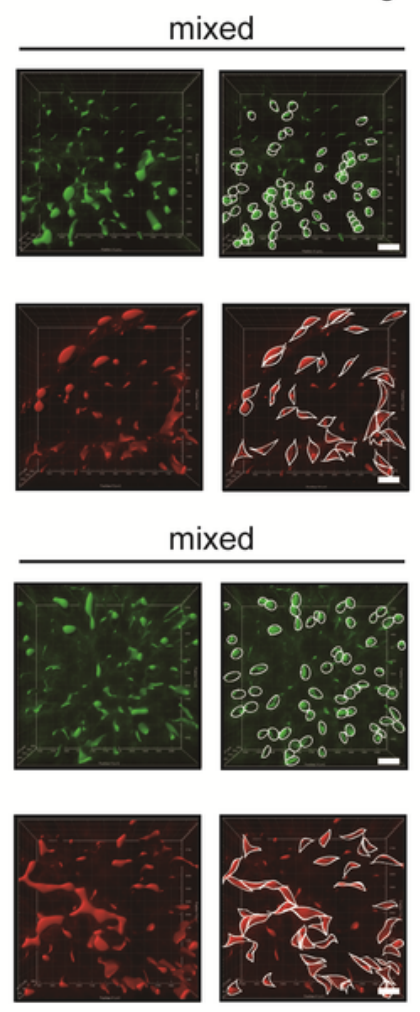

$f$

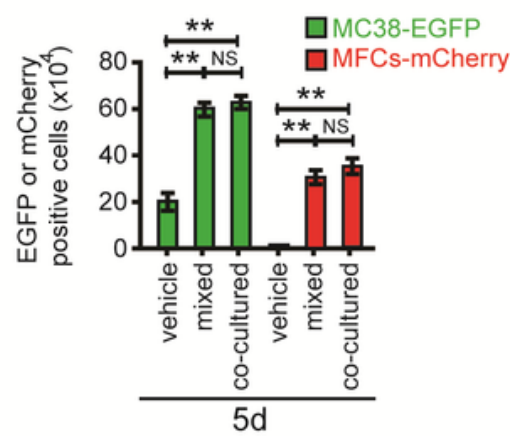

g

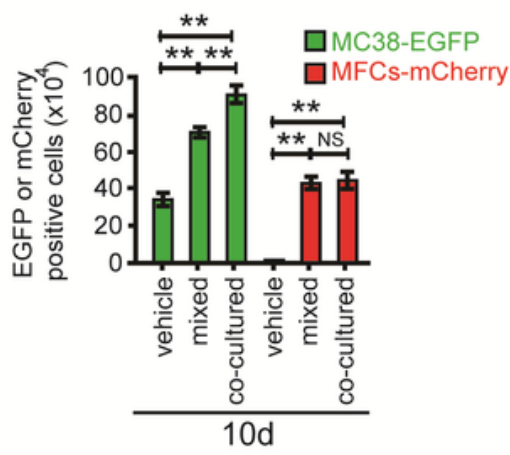

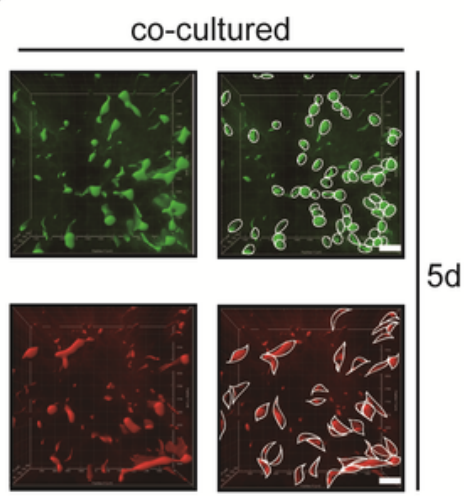

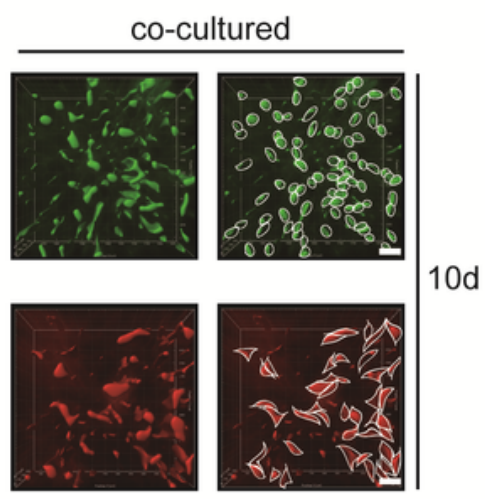

Figure 5

The co-metastasis of CRC cells and MFCs in DLNs. a. The schedule of mouse footpad injection. The mice were injected with co-cultured or mixed MC38 cells tagged with EGFP and MFCs tagged with mCherry. MC38 cells alone was set as the vehicle group. b, c. Photos of DLNs obtained from mice at $5 \mathrm{~d}$ and $10 \mathrm{~d}$ 
(b). The statistical results were shown below (c). d, e. 3D images of EGFP-tagged MC38 cells and mCherry-tagged MFCs in cleared DLNs at $5 \mathrm{~d}(\mathrm{~d})$ and $10 \mathrm{~d}(\mathrm{e})$. A randomly selected 3D image (500 $\mu \mathrm{m}-\mathrm{X}$ axis $\times 500 \mu \mathrm{m}-Y$ axis $\times 100 \mu \mathrm{m}-Z$ axis) was taken out and shown here. The number of MC38 cells or MFCs were quantified according to the 3D images. $\mathrm{f}, \mathrm{g}$. The statistical results of 3D images of EGFPtagged MC38 cells and mCherry-tagged MFCs in cleared DLNs at $5 \mathrm{~d}(\mathrm{f})$ and $10 \mathrm{~d}(\mathrm{~g})$. Scale bars: $10 \mu \mathrm{m}$. All experiments were repeated at least three times.

a

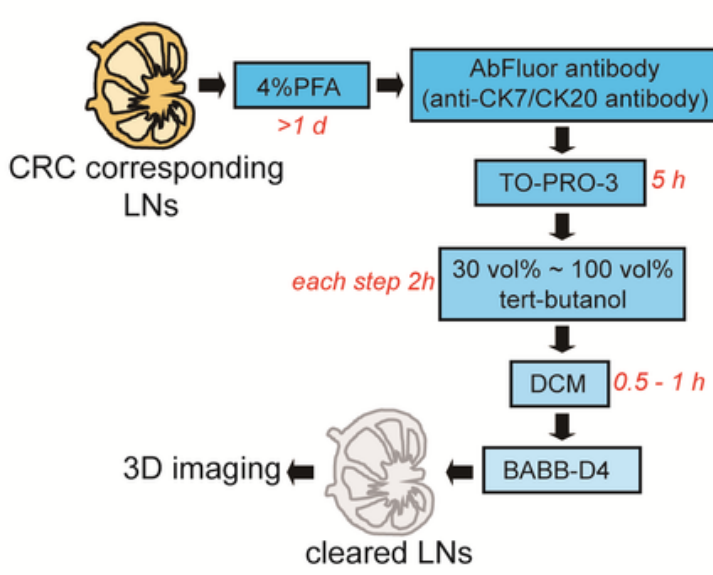

b

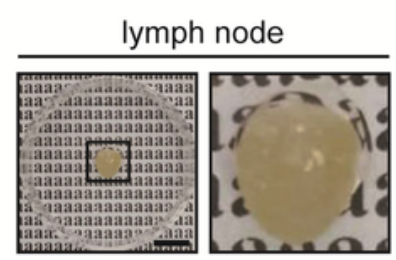

cleared lymph node

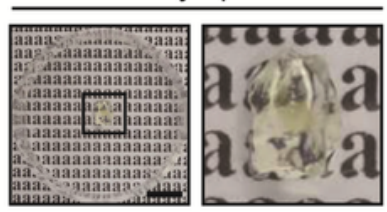

C
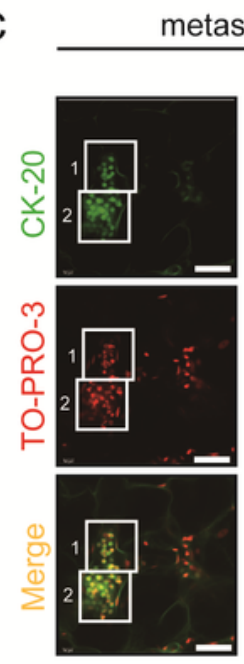

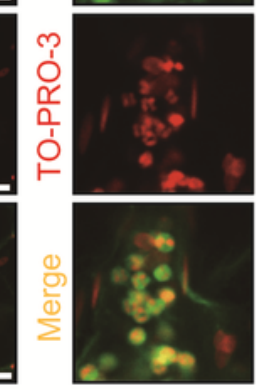

e

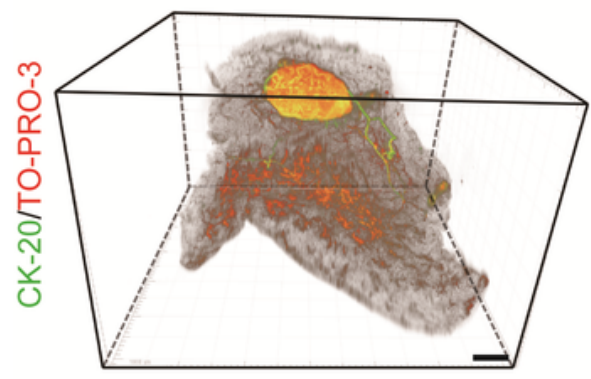

d
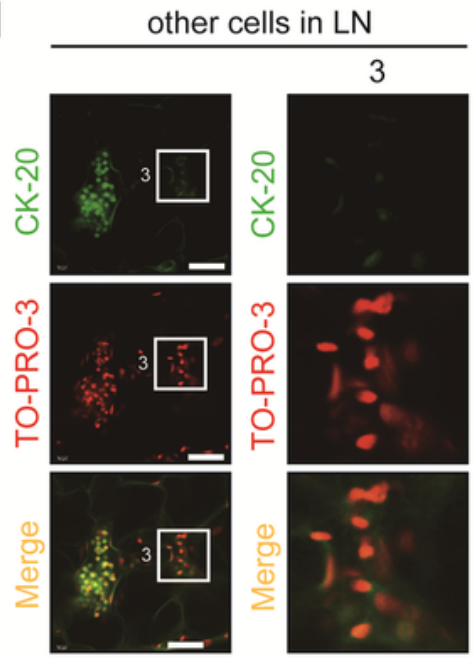

f

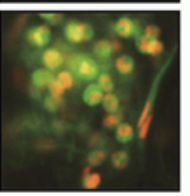

whole-mount tissue 3D imaging
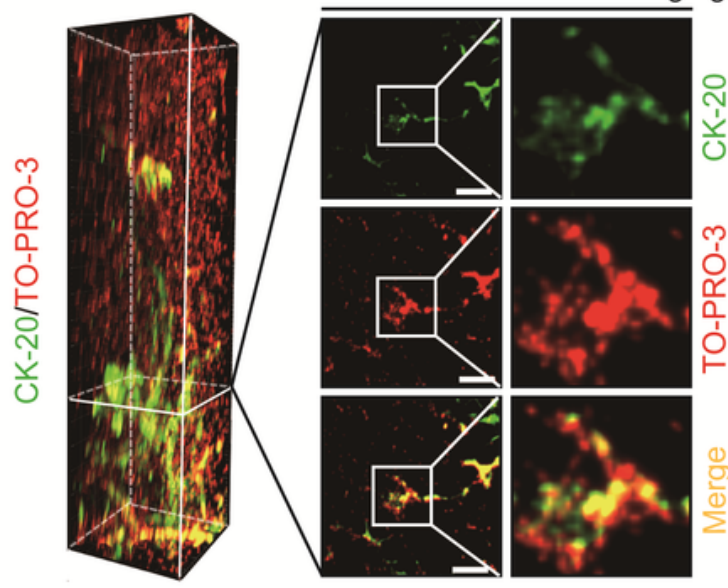

Figure 6 
The whole-mount tissue 3D imaging of mesenteric LN tissues obtained from clinical CRC patients. a. The uDISCO protocols of clinical CRC corresponding mesenteric LN tissues. b. Photos of mesenteric LNs before and after UDISCO clearing. Scale bars: $1200 \mu \mathrm{m}$. c, d. Photos of metastatic CRC cells and other cells detected by laser confocal microscope. e. 3D imaging of a $L N$ tissue which was collected from a 70year-old man. $\mathrm{f}$. Cross-section photos of invaded CRC cells in above LN tissue.

\section{Supplementary Files}

This is a list of supplementary files associated with this preprint. Click to download.

- Supplementarymaterial.doc

- SupplementaryVideo1.mp4

- SupplementaryVideo2.mp4

- SupplementaryVideo3.mp4

- SupplementaryVideo4.mp4 